\title{
Modeling of Daptomycin Release from Medium-Dose Daptomycin-Xylitol-Loaded PMMA Bone Cements
}

\author{
Ali Salehi' ${ }^{1}$, Gladius Lewis ${ }^{1}$, Ashley Cox Parker'², Warren 0. Haggard ${ }^{2}$ \\ ${ }^{1}$ Department of Mechanical Engineering, The University of Memphis, Memphis, USA \\ ${ }^{2}$ Department of Biomedical Engineering, The University of Memphis, Memphis, USA \\ Email: ${ }^{*}$ glewis@memphis.edu
}

Received 31 March 2014; revised 5 May 2014; accepted 13 May 2014

Copyright (C) 2014 by authors and Scientific Research Publishing Inc.

This work is licensed under the Creative Commons Attribution International License (CC BY).

http://creativecommons.org/licenses/by/4.0/

(c) (i) Open Access

\section{Abstract}

Antibiotic-loaded poly (methyl methacrylate) bone cements (ALPBCs) are widely used as an agent to decrease the occurrence of periprosthetic joint infection (PJI). Most often, the antibiotic used in an ALPBC is gentamicin, tobramycin, or vancomycin. In many recent clinical studies, it has been reported that the pathogens that commonly present in PIJ are becoming resistant to these antibiotics. As such, a new generation of antibiotics is emerging, among which is daptomycin. Literature reports with a clinically relevant medium-dose daptomycin-loaded cement show that the daptomycin release rate from cylindrical specimens is low. Incorporation of a poragen, such as dextrose, glycine, or particulate xylitol, into the cement powder has been shown to be an effective way to increase daptomycin release rate. There are, however, no studies on modeling of daptomycin release from specimens of either a daptomycin-loaded cement or a daptomycin-poragen-loaded cement. In the present work, we determine the profiles of daptomycin release from cylindrical medium-dose daptomycin-xylitol-loaded cement specimens, as a function of the particulate xylitol loading. We used these results and relationships that have been shown to model antibiotic release from ALPBC specimens to obtain the best-fit relationship for the present cements. Through this approach, we demonstrated that, regardless of the xylitol loading, the daptomycin release profile is a mixture of initial burst followed by a slow Fickian diffusion.

\section{Keywords}

Poly (Methyl Methacrylate) Bone Cement, Daptomycin, Xylitol, Elution

\footnotetext{
${ }^{*}$ Corresponding author.
} 


\section{Introduction}

Although there is no consensus on the incidence of periprosthetic joint infection (PJI) in total joint replacements, with values ranging from $<1 \%$ for primary cases [1] to $>40 \%$ for revisions [2], it is universally acknowledged that PJI is a major, if not intractable, problem. Specifically, PJI is a very serious complication for the patient (necessitating revisions, in some cases [3] [4]) and for the healthcare system (for example, in 2009, in the United States, the cost of surgical treatment of an infected arthroplasty ranged from $\sim \$ 94,000$ to $\sim \$ 144,000$ [5] [6]). To reduce the likelihood of PJI, antibiotic-loaded poly (methyl methacrylate) (PMMA) bone cements (ALPBCs) are widely used as a prophylaxis agent in primary cases or a treatment option in certain types of revision cases, such as second-stage reconstructions [7] [8]. For the former cases, there are a large number of approved low-dose commercially-available brands of these cements [7] [8]. A low-dose is defined as antibiotic loading of $1 \mathrm{~g}$ of antibiotic mixed with $40 \mathrm{~g}$ of dry cement powder [7]. For applicable implant revision cases, medium-dose ALPBC formulations are prepared by the attending orthopaedic surgeon or a designated member of the surgical team [9]. A medium-dose is herein defined as antibiotic loading of between $1 \mathrm{~g}$ and $4 \mathrm{~g}$ of antibiotic per $40 \mathrm{~g}$ of dry cement powder. In either type of case, it is very common that the antibiotic mixed with the cement powder is gentamicin, tobramycin, vancomycin, or some combination of these [7] [8]. Clinical studies are indicating that the pathogens that commonly present with PJI, such as methicillin-resistant Staphylococcus aureus and Staphylococcus epidermidis, are developing resistance to these antibiotics [10] [11]. As such, a new generation of antibiotics is emerging, among which are ceftobiprole, dalbavancin, daptomycin, telavancin, and tigecycline [12]-[19]. Of these, only daptomycin has been incorporated in a PMMA bone cement and reported in some in vitro studies [20]-[25] and only daptomycin and telavancin have been used in PJI treatment [18] [19].

Results of the few studies on the release of daptomycin from cylindrical specimens of medium-dose daptomycin-loaded PMMA bone cement show that 1) it is characterized by an initial burst of the antibiotic within 24 $\mathrm{h}$, followed by low amount of sustained elution; and 2) the released daptomycin is effective against S. aureus [21] [25]. There are a plethora of methods to increase the rate of an antibiotic from ALPBC specimens, such as incorporation of a poragen (for example, particulate xylitol and glycine) in the cement powder, low-level ultrsonication, and mechanical mixing [26]-[32]. The literature on enhancement of release of daptomycin from daptomycin-loaded PMMA bone cement specimens is limited, with the only method used being incorporation of particulate xylitol in the cement powder [24] [25] [33]. It has also been shown that daptomycin released from cylindrical daptomycin-particulate xylitol-PMMA bone cement specimens is effective against $S$. aureus [25]. Thus, the daptomycin-particulate xylitol-PMMA bone cement system is clinically relevant. While studies on modeling of the release of gentamicin, tobramycin, and vancomycin from ALPBC cylinders abound [26] [34] [35], there are none on the daptomycin-particulate xylitol-PMMA bone cement system. The purpose of the present study was to propose an applicable daptomycin release mechanism for this system.

\section{Materials and Methods}

\subsection{Materials}

The materials used were a commercially-available brand of a high-viscosity PMMA bone cement (Orthoset ${ }^{\circledR} 1$ Radiopaque Bone Cement; Wright Medical Technology, Arlington, TN, USA), daptomycin (Cubicin ${ }^{\circledR}$; Cubist Pharmaceuticals, Lexington, MA, USA), and particulate xylitol (XyloSweet ${ }^{\circledR}$; XLEAR, Orem, UT, USA). The daptomycin loading used (3.3 wt/wt\%; equivalent to $1.36 \mathrm{~g}$ per $40 \mathrm{~g}$ of dry cement powder) was determined from our previous work on daptomycin-loaded Orthoset ${ }^{\circledR} 1$,based on a balance between three cement properties, namely, daptomycin release rate, an index of inhibition of the daptomycin eluate against $S$. aureus, and fatigue limit [21].

\subsection{Study Design}

The study design comprised four parts: 1) fabrication of four groups of specimens, one not loaded with the xylitol (XYL-00 group) and three having different amounts of xylitol per $40 \mathrm{~g}$ of dry cement powder; namely, $0.7 \mathrm{~g}$ (XYL-07 group), $1.4 \mathrm{~g}$ (XYL-14 group), and $2.7 \mathrm{~g}$ (XYL-27 group), corresponding to xylitol loadings of 1.66, 3.27 , and $6.13 \mathrm{wt} / \mathrm{wt} \%$, respectively. The range of xylitol loading used was the same as that in our previous study in which we computed what we designated an "appropriate xylitol loading” being the loading that yields a cement that has an optimal combination of the seven properties we determined, namely, daptomycin release rate; 
an index of inhibition of the daptomycin eluate against S. aureus; coefficient of diffusion of egress of daptomycin; coefficient of diffusion of ingress of $1 \mathrm{X}$ phosphate buffered saline (PBS) solution, at $37^{\circ} \mathrm{C}$; polymerization rate, at $37^{\circ} \mathrm{C}$; fracture toughness; and fatigue limit [25]; 2) for each group, determination of the daptomycin release profile (amount of released daptomycin-versus-length of time of specimen in $1 \mathrm{X}$ PBS solution, at $37^{\circ} \mathrm{C}$ ); 3 ) for each group, determination of the morphology of the specimens at the end of the daptomycin release studies; and 4) for each group, determination of the applicable daptomycin release mechanism by fitting four antibiotic elution kinetics models to the release profile.

\subsection{Specimen Fabrication}

The protocols used in the preparing the cement specimens were the same as presented in our previous work [25]. In essence, 1) the cement powder, daptomycin, and xylitol were mixed using a commercially-available cement powder mixer (OmoMix ${ }^{\circledR}$; Tecres SpA, Verona, Italy) and then the mixture was vacuum-mixed with the liquid monomer of the cement $(18.37 \mathrm{~mL})$; 2$)$ the resulting cement dough was injected into a silicone mold that has four cells, with each cell having internal configuration and dimensions of a solid cylindrical dog-bone, whose dimensions conformed to those stipulated in ASTM F2118 [36]; and 3) after curing in the mold, in ambient laboratory air, for 20 minutes, the specimens (Figure 1 ) were removed, lightly sanded, and then inspected for flaws. Protocols for inspecting the fabricated specimens, selecting acceptable specimens, and conditioning the acceptable specimens prior to the daptomycin release testing, were the same as described in our previous work [21] [25].

\subsection{Determination of Cement Properties}

For each study group, from among the $78 \%$ - $89 \%$ of the specimens found acceptable, three were selected at random for the daptomycin release tests. Each specimen was weighed using an analytic balance (Model A-160; Fisher Scientific, Inc., Fairlawn, NJ, USA) and then placed in a 15-mL centrifuge tube containing $10 \mathrm{~mL} 1 \mathrm{X}$ PBS. The tube was then placed in an incubator at $37^{\circ} \mathrm{C}$. At time points (t) of $1,2,5,7,10,14,21$, and 28 days, the tube was shaken and four 1-mL aliquots were taken, placed in microcentrifuge tubes, and frozen $\left(\right.$ at $\left.-80^{\circ} \mathrm{C}\right)$ until testing. At each time point, the remaining PBS was discarded and a fresh $10 \mathrm{~mL} 1 \mathrm{X}$ PBS was added. The eluate volume was chosen such that low concentrations could still be measured through day 28, while allowing the test specimen to be completely submerged. Theamount of daptomycin in each aliquot was measured using a protocol given by Richelsoph et al. [37] and high-performance liquid chromatography (Varian Prostar; Varian, Inc., Palo Alto, CA, USA), at $530 \mathrm{~nm}$. The daptomycin release amount was calculated as the mass of daptomycin in the eluate divided by the product of the mass of the test specimen and the volume of 1X PBS used [21] [25]. The release results were presented as current daptomycin release amount versus $t$ and cumulative daptomycin release amount $(\mathrm{M})$ versus $\mathrm{t}$.

\subsection{Morphology Determination}

After the daptomycin release tests, test discs (nominal diameter and thickness $=5.0 \mathrm{~mm}$ and $3.0 \mathrm{~mm}$, respectively) were cut from the test specimens (three per group). The morphology of each disc was observed using an environmental scanning electron microscope (Model XL30; Philips, Eindhoven, Netherlands), at an accelerating voltage of $15 \mathrm{kV}$ and a magnification of 200.

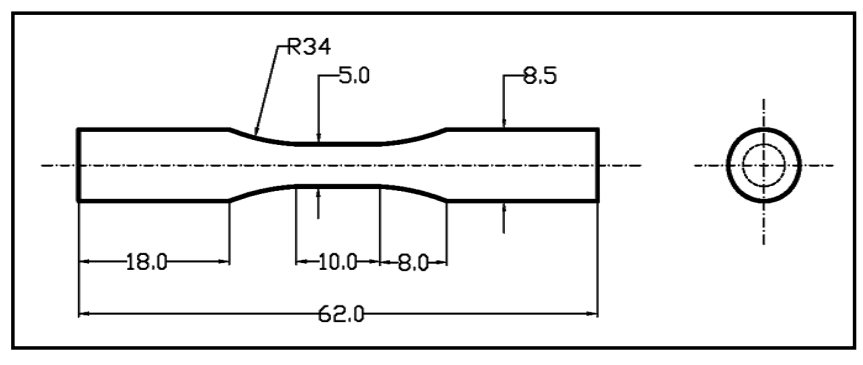

All dimensions are in mm.

Figure 1. Dimensioned drawing of the test specimen. 


\subsection{Modeling Equations}

For each of the study groups, four kinetics equations that have been widely used to gain insight into the mechanism(s) involved in the release of an antibiotic from ALPBC specimens were fitted to the $M_{t}$ results. These equations, in order, are those given by Korsmeyer et al. [38], Lindner and Lippold [39], Frutos et al. [35], and Hesaraki et al. [40]; namely

$$
\begin{gathered}
M_{t} / M_{\infty}=k_{1} t_{1}^{n} \\
M_{t} / M_{\infty}=b+k_{2} t_{2}^{n} \\
M_{t} / M_{\infty}=A+B\left(1-e_{3}^{-n t}\right)+C t^{0.5} \\
M_{t} / M_{\infty}=k_{3}\left[1-e^{-(t / \tau) d}\right]
\end{gathered}
$$

The definitions of the coefficients $\left(k_{1}, n_{1}, b, k_{2}, n_{2}, A, B, n_{3}, C, k_{3}, \tau\right.$, and $\left.d\right)$ in Equations (1)-(4) are given in Table 1, and their values were determined using a nonlinear least squares method contained in a commerciallyavailable software package (Origin Pro 8.6; Origin Lab Corp, Northampton, MA, USA).

\section{Results}

Following daptomycin release, the morphology of the cement specimens may be described as porous (Figure 2).

Although each of the release kinetics models provided a very good fit to the experimental M-versus-t results (that is, in each case, adjusted $R^{2}>0.96$ - see Table 2), Equations (1) and (4) suffer from the fact that neither contains a term that could be associated with initial burst of the daptomycin (a phenomenon that is in clear display-see Figure 3(a) and Figure 3(b)). With respect to Equation (3), the best-fit value of the release time exponent $\left(n_{3}\right)$ has exactly the same value for all the study groups, suggesting that the elution mechanism is a constant rate of release. This, clearly, is not the case. Thus, we are left with Equation (2). We note one similarity and three differences in the best-fit values of the constants in Equation (2) when the xylitol-containing cements (taken as a group) are compared to the cement that did not contain xylitol. The similarity is that, for each cement, the best-fit value of $n_{2}$ is $\ll 0.5$. The three differences are that there is a very substantial increase (mean of $\sim 1025 \%$ ) in the best-fit value of $b$, a marked decrease (mean of $\sim 50 \%$ ) in the best-fit value of $k_{2}$, and a very large increase (mean of $\sim 94 \%$ ) in the best-fit value of $n_{2}$. The consequences of these differences are seen in an improvement in the daptomycin release profile in cements that contained xylitol compared to the one that did not; specifically, the time taken for the daptomycin release to be completed was $\sim 18$ days when the xylitol loading was zero but almost doubled (to $\sim 35$ days) when the loading was $1.66 \mathrm{wt} / \mathrm{wt} \%$ or higher (Figure 3(a)).

\section{Discussion}

Antibiotic-loaded PMMA bone cements are widely used in the treatment of PJI [7] [8]. Typically, gentamicin, tobramycin, or vancomycin is the antibiotic used in these cements [7] [8]. The pathogens that are commonly found in PJI cases are becoming increasing resistant to these antibiotics [10] [11]; as such, various newer antibiotics are now becoming available, examples being daptomycin and telavancin [12] [14] [15] [18] [19]. The literature on the use of daptomycin to treat PJI [18] and on in vitro studies of daptomycin-loaded PMMA bone

Table 1. Definitions of constants in Equations (1)-(4).

Constant
$k_{1} ; k_{2} \quad$ Each constant is related to the characteristics of the macromolecular network of the cement matrix and the daptomycin
$n_{1} ; n_{2} ; C ; d \quad$ An exponent that denotes that the mechanism of release of the daptomycin from the cement matrix is a diffusion process
$b ; A$
$\begin{gathered}\text { E } ; n_{3} \\ k_{3}\end{gathered} \quad$ Each is a term that characterizes the initial burst of the daptomycin from the cement
$\tau$


Table 2. Results of the daptomycin elution kinetics model curve fitting: mean alues of coefficients.

\begin{tabular}{|c|c|c|}
\hline Cement & Mean values of coefficients & Adj $R^{2}$ \\
\hline \multicolumn{3}{|c|}{ Equation (1) } \\
\hline XYL-00 & $k_{1}=0.4672 ; \quad n_{1}=0.05133$ & 0.9989 \\
\hline XYL-07 & $k_{1}=0.2257 ; \quad n_{1}=0.10025$ & 0.9691 \\
\hline XYL-14 & $k_{1}=0.2773 ; \quad n_{1}=0.08630$ & 0.9953 \\
\hline XYL-27 & $k_{1}=0.2225 ; \quad n_{1}=0.10056$ & 0.9809 \\
\hline \multicolumn{3}{|c|}{ Equation (2) } \\
\hline XYL-00 & $k_{2}=0.4672 ; \quad n_{2}=0.05133 ; \quad b=0.62 \times 10^{-4}$ & 0.9988 \\
\hline XYL-07 & $k_{2}=0.2253 ; \quad n_{2}=0.10034 ; \quad b=7.87 \times 10^{-4}$ & 0.9678 \\
\hline XYL-14 & $k_{2}=0.2770 ; \quad n_{2}=0.08634 ; \quad b=4.18 \times 10^{-4}$ & 0.9951 \\
\hline XYL-27 & $k_{2}=0.2220 ; \quad n_{2}=0.10067 ; \quad b=8.87 \times 10^{-4}$ & 0.9801 \\
\hline \multicolumn{3}{|c|}{ Equation (3) } \\
\hline XYL-00 & $A=3.64 \times 10^{-16} ; \quad B=0.8184 ; C=1.22 \times 10^{-4} ; \quad n_{3}=1$ & 0.9988 \\
\hline XYL-07 & $A=2.54 \times 10^{-11} ; \quad B=0.6660 ; C=2.22 \times 10^{-4} ; \quad n_{3}=1$ & 0.9704 \\
\hline XYL-14 & $A=3.83 \times 10^{-11} ; \quad B=0.7061 ; C=1.95 \times 10^{-4} ; \quad n_{3}=1$ & 0.9970 \\
\hline XYL-27 & $A=2.53 \times 10^{-10} ; \quad B=0.6579 ; C=2.21 \times 10^{-4} ; \quad n_{3}=1$ & 0.9844 \\
\hline \multicolumn{3}{|c|}{ Equation (4) } \\
\hline XYL-00 & $k_{3}=2.663 ; \quad \tau=3.75 \times 10^{11} ; \quad d=0.06379$ & 0.9988 \\
\hline XYL-07 & $k_{3}=4.131 ; \quad \tau=2.53 \times 10^{11} ; \quad d=0.11260$ & 0.9676 \\
\hline XYL-14 & $k_{3}=3.635 ; \quad \tau=2.72 \times 10^{11} ; \quad d=0.09905$ & 0.9950 \\
\hline XYL-27 & $k_{3}=4.090 ; \quad \tau=2.44 \times 10^{11} ; \quad d=0.11290$ & 0.9798 \\
\hline
\end{tabular}

${ }^{\mathrm{a} C}$ Coefficient of multiple determination, adjusted/corrected for the degrees of freedom of the equation.

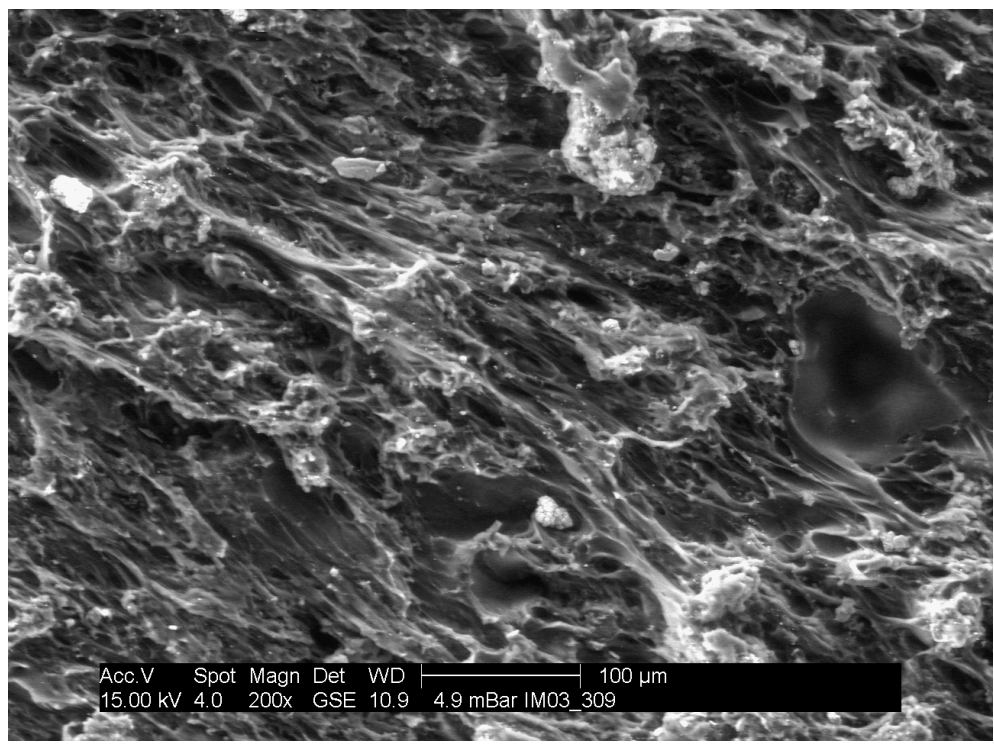

Figure 2. Morphology of a representative cement specimen, following release of daptomycin. Note the white specks (mostly, $\mathrm{BaSO}_{4}$ particles) layered on the underlying porous regions (dark elongated ridges). 


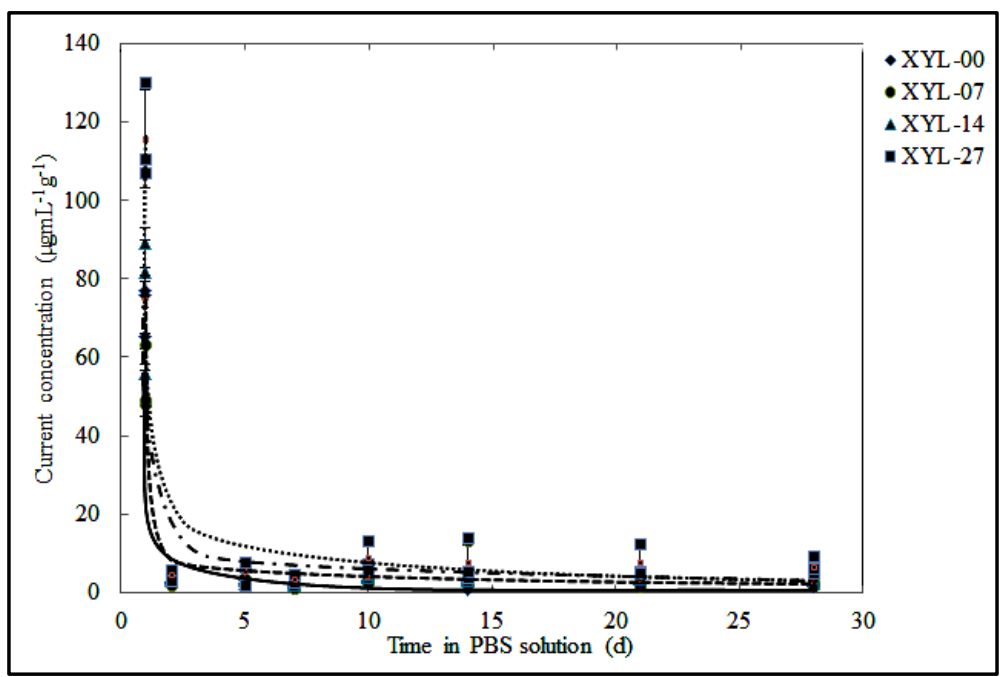

(a)

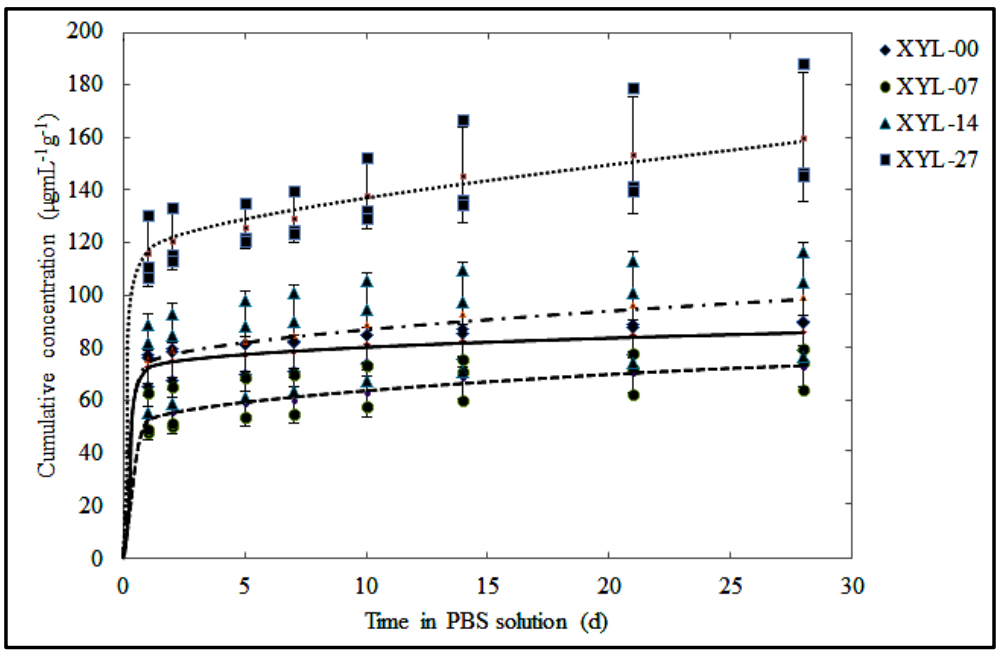

(b)

Figure 3. The daptomycin release profiles in current amount (a) and cumulative amount (b). Study sets were: no xylitol (XYL-00), 0.7 wt/wt\% xylitol (XYL-07), $1.4 \mathrm{wt} / \mathrm{wt} \%$ xylitol (XYL-14), and $2.7 \mathrm{wt} / \mathrm{wt} \%$ xylitol (XYL-27).

cement is very limited [20]-[25]. Onlya few in vitro studies have been reported on the increase of the rate of release of daptomycin from PMMA bone cement specimens, this being achieved by mixing a poragen (particulate xylitol) with the cement powder [24] [25]. There are no reports on modeling of daptomycin release from daptomycin-loaded PMMA bone cement specimens. In the present study, our purpose was to perform this modeling in the case of daptomycin-xylitol-loaded PMMA bone cement cylinders, as a function of the xylitol loading.

In the literature, there is no consensus regarding the release mechanism(s) of an antibiotic from an ALPBC specimen, with some proposed ones being a pure surface phenomenon, diffusion of the antibiotic through the solid part of the cement matrix, and diffusion of the antibiotic through the channels of cracks and voids in the cement matrix [42] [43]. In support of these proposals, a number of antibiotic release kinetics models have been presented in the literature [35] [38]-[40] [44], a small sample of which was tested in the present work. Our results led us to postulate that the release of daptomycin from cylindrical specimens of either a daptomycin-loaded cement or a daptomycin-xylitol-loaded cement involves 1) initial burst of the daptomycin from either the surface or a zone close to the surface of the specimen and 2) Fickian diffusion of the daptomycin through pores in the specimen matrix. The latter part of this postulate is based on the fact that, for each cement, $n_{2}<0.5$ [44].

We suggest that the very large increase in the best-fit value of $n_{2}$ for a cement that contained xylitol (com- 
pared to the value for the cement that did not contain xylitol) reflects an increase in porosity of the former cement specimen that is caused by the xylitol addition. This phenomenon, in conjunction with the marked decrease in the best-fit value of $k_{2}$, accounts for the increased daptomycin release rate from the daptomycin-xylitol-loaded cement specimens.

To the best of our knowledge, only Nugent et al. [27] have reported on the influence of xylitol on antibiotic release from ALPBC cylindrical specimens; thus, it is germane to compare and contrast our study to theirs. Nugent et al. used Surgical Simplex ${ }^{\circledR} \mathrm{P}$ cement (a medium-viscosity cement [45]), tobramycin, amounts of xylitol that ranged from 0 to $16 \mathrm{~g}$ per $1 \mathrm{~g}$ of tobramycin and $40 \mathrm{~g}$ of dry cement powder; hand mixed the final powder mixture with the cement liquid; determined the amount of tobramycin released as functions of the xylitol loading and the time of immersion of the specimens in $1 \mathrm{X} \mathrm{PBS}$, at $37^{\circ} \mathrm{C}(\mathrm{T} ; 0 \leq \mathrm{T} \leq 30 \mathrm{~d})$; but did not provide any information on modeling of tobramycin release. In the present study, we used Orthoset ${ }^{\circledR} 1$ (a high-viscosity brand [45]), daptomycin, amounts of xylitol that ranged from 0 to $2.7 \mathrm{~g}$ per $1.36 \mathrm{~g}$ of daptomycin and $40 \mathrm{~g}$ of dry cement powder; vacuum mixed the final powder mixture with the cement liquid; determined the amount of daptomycin released as a function of $\mathrm{T}(0 \leq \mathrm{T} \leq 28 \mathrm{~d})$; and modeled the daptomycin release. In both studies, at a comparable range of xylitol loading, the antibiotic release rate increased with increase in xylitol loading.

Our study has a number of limitations. First, we used a poragen (particulate xylitol) for which there are no reports on its use as a constituent of an orthopaedic material. However, there are reports of xylitol enhancing daptomycin release from PMMA pellets in a preclinical rabbit model of chronic, postsurgical osteomyelitis [33] and beneficial effects of dietary xylitol against weakening of bone in aged animal models [46]. Thus, the expectation is that it will be safe to use xylitol-containing ALPBCs as a prophylaxis agent against PJI. Second, in the interest of time and to keep the study cost reasonable, we used only one cement brand, but we do not expect cement brand to greatly affect the trends found in the present work. Third, we recognize that in vivo, there are parameters, such as blood clot and protein adsorption, that are important vis a vis the diffusion of an antibiotic from ALPBCs. However, the solution used in the present daptomycin release determination protocol (namely, $1 \mathrm{X}$ PBS, at $37^{\circ} \mathrm{C}$ ) is both well-established and appropriate [27] [34] [47]. We submit, therefore, that none of these limitations undermines the validity of our study findings.

\section{Conclusion}

We conclude that 1) release of daptomycin from cylindrical daptomycin-loaded PMMA bone cement specimens may be modeled by an expression that accounts for initial burst of the antibiotic followed by its slow and low release controlled by Fickian diffusion; and 2) this mechanism is unchanged when xylitol is blended with the daptomycin and the dry cement powder (over the range of 0.7 - $2.7 \mathrm{~g}$ xylitol per $1.36 \mathrm{~g}$ daptomycin and $40 \mathrm{~g}$ cement powder) although there are marked increases in the model constants associated with both the initial burst and the diffusion.

\section{Acknowledgements}

We thank Cubist Pharmaceuticals, for funding the work and generous donation of the daptomycin; Wright Medical Technology, for their help in obtaining Orthoset ${ }^{\circledR} 1$ bone cement; and Dr. Si Janna, for fabricating the test specimens.

\section{References}

[1] Hirakawa, K., Stulberg, B.N., Wilde, A.H., Bauer, T.W. and Secic, M. (1998) Results of 2-Stage Reimplantation for Infected Total Knee Arthroplasty. The Journal of Arthroplasty, 13, 22-28. http://dx.doi.org/10.1016/S0883-5403(98)90071-7

[2] Trampuz, A. and Zimmerlt, W. (2005) Prosthetic Joint Infections: Update in Diagnosis and Treatment. Swiss Medical Weekly, 135, 243-251.

[3] Lentino, J.R. (2003) Prosthetic Joint Infections: Bane of Orthopedists, Challenge for Infectious Disease Specialists. Clinical Infectious Diseases, 36, 1157-1161. http://dx.doi.org/10.1086/374554

[4] Parvizi, J., Adeli, B., Zmistowski, B., Restrepo, C. and Greenwald, A.S. (2012) Management of Periprosthetic Joint Infection: The Current Knowledge. The Journal of Bone \& Joint Surgery, 94, e104(1-9).

[5] Parvizi, J., Pawasarat, I.M., Azzam, K.A., Joshi, A., Hansen, E.N. and Bozic, K.J. (2010) Periprosthetic Joint Infection: The Economic Impact of Methicillin-Resistant Infections. The Journal of Arthroplasty, 25, 103-107. 
http://dx.doi.org/10.1016/j.arth.2010.04.011

[6] Kurtz, S.M., Lau, E., Watson, H., Schmer, J.K. and Parvizi, J. (2012) Economic Burden of Periprosthetic Joint Infection in the United States. The Journal of Arthroplasty, 27, 61-65e1.

[7] Jiranek, W.A., Hanssen, A.D. and Greenwald, A.S. (2006) Antibiotic-Loaded Bone Cement for Infection Prophylaxis in Total Joint Replacement. The Journal of Bone \& Joint Surgery, 88, 2487-2500. http://dx.doi.org/10.2106/JBJS.E.01126

[8] Parvizi, J., Saleh, K.J., Ragland, P.S., Pour, A.E. and Mont, M.A. (2008) Efficacy of Antibiotic-Impregnated Cement in Total Hip Replacement: A Meta-Analysis. Acta Orthopaedica, 79, 335-3341. http://dx.doi.org/10.1080/17453670710015229

[9] Meyer, J., Piller, G., Spiegel, C.A., Hetzel, S. and Squire, M. (2011) Vacuum-Mixing Significantly Changes Antibiotic Elution Characteristics of Commercially Available Antibiotic-Impregnated Bone Cements. The Journal of Bone \& Joint Surgery, 93, 2049-2056. http://dx.doi.org/10.2106/JBJS.J.01777

[10] Wenzel, R.P. (2004) The Antibiotic Pipeline-Challenges, Costs, and Values. The New England Journal of Medicine, 351, 523-526. http://dx.doi.org/10.1056/NEJMp048093

[11] Campoccia, D., Montanaro, L. and Arciola, C.R. (2006) The Significance of Infection Related to Orthopedic Devices and Issues of Antibiotic Resistance. Biomaterial, 27, 2331-2339. http://dx.doi.org/10.1016/j.biomaterials.2005.11.044

[12] Higgins, D.L., Chang, R., Debabov, D.V., Leung, J., Wu, T., Krause, K.M., Sandvik, E., Hubbard, J.M., Kaniga, K., Schmidt Jr., D.E., Gao, Q., Cass, R.T., Karr, D.E., Benton, B.M. and Humphrey, P.P. (2005) Telavancin, a Multifunctional Lipoglycopeptide, Disrupts Both Cell Wall Synthesis and Cell Membrane Integrity in Methicillin-Resistant Staphylococcus aureus. Antimicrob Agent Chemotherapy, 49, 1127-1134. http://dx.doi.org/10.1128/AAC.49.3.1127-1134.2005

[13] Doan, T.L., Fung, H.B., Mehta, D. and Riska, P.F. (2006) Tigecycline: A Glycocycline Antimicrobial Agent. Clinical Therapy, 28, 1079-1086. http://dx.doi.org/10.1016/j.clinthera.2006.08.011

[14] Sauermann, R., Rothenburger, M., Graninger, W. and Joukhadar, C. (2008) Daptomycin: A Review 4 Years after First Approval. Pharmacology, 81, 79-91. http://dx.doi.org/10.1159/000109868

[15] Song, J.-H. (2008) What's New on the Antimicrobial Horizon? International Journal of Antimicrobial Agents, 32, S207-S213. http://dx.doi.org/10.1016/S0924-8579(09)70004-4

[16] Huang, V., Cheung, C.M., Kaatz, G.W. and Rybak, M.J. (2010) Evaluation of Dalbavancin, Tigecycline, Minocycline, Tetracycline, Teicoplanin and Vancomycin against Community-Associated and Multidrug-Resistant Hospital-Associated Methicillin-Resistant Staphylococcus aureus. International Journal of Antimicrobial Agents, 35, 25-29. http://dx.doi.org/10.1016/j.ijantimicag.2009.08.020

[17] Cai, Y., Wang, R., Liang, B., Bai, N. and Liu, Y. (2011) Systematic Review and Meta-Analysis of the Effectiveness and Safety of Tigecycline for Treatment of Infectious Disease. Antimicrobial Agents and Chemotherapy, 55, 11621172. http://dx.doi.org/10.1128/AAC.01402-10

[18] Licitra, C.M., Crespo, A., Licitra, D. and Wallis-Crespo, M.C. (2011) Daptomycin for the Treatment of Osteomyelitis and Prosthetic Joint Infection: Retrospective Analysis of Efficacy and Safety in an Outpatient Infusion Center. The Internet Journal of Infectious Diseases, 9, Published Online. http://dx.doi.org/10.5580/215c

[19] Kaushal, R. and Hassoun, A. (2012) Successful Treatment of Methicillin-Resistant Staphylococcus epidermidis Prosthetic Joint Infection with Telavancin. Journal of Antimicrobial Chemotherapy, 67, 2052-2053. http://dx.doi.org/10.1093/jac/dks165

[20] Rouse, M.S., Piper, K.E., Jacobson, M., Jacofsky, D.J., Steckelberg, J.M. and Patel, R. (2006) Daptomycin Treatment of Staphylococcus aureus Experimental Chronic Osteomyelitis. Journal of Antimicrobial Chemotherapy, 57, 301-305. http://dx.doi.org/10.1093/jac/dki435

[21] Lewis, G., Brooks, J.L., Courtney, H.S., Li, Y. and Haggard, W.O. (2010) An Approach for Determining Antibiotic Loading for a Physician-Directed Antibiotic-Loaded PMMA Bone Cement Formulation. Clinical Orthopaedics and Related Research, 468, 2092-2100. http://dx.doi.org/10.1007/s11999-010-1281-0

[22] Chang, Y., Chen, W.C., Hsieh, P.H., Chen, D.W., Lee, M.S., Shih, H.N. and Ueng, S.W.N. (2011) In Vitro Activities of Daptomycin-, Vancomycin-, and Teicoplanin-Loaded Polymethylmethacrylate against Methicillin-Susceptible, Methicillin-Resistant, and Vancomycin-Intermediate Strains of Staphylococcus aureus. Antimicrobial Agents and Chemotherapy, 55, 5480-5484. http://dx.doi.org/10.1128/AAC.05312-11

[23] Kaplan, L., Kurdzeil, M., Baker, K.C. and Verner, J. (2012) Characterization of Daptomycin-Loaded Antibiotic Cement. Orthopedics, 35, e503-e509. http://dx.doi.org/10.3928/01477447-20120327-19

[24] McLaren, A.C., McLaren, S.G. and Smeltzer, M. (2006) Xylitol and Glycine Fillers Increase Permeability of PMMA to Enhance Elution of Daptomycin. Clinical Orthopaedics and Related Research, 451, 25-28.

[25] Salehi, A., Parker, A.C., Lewis, G., Courtney, H.S. and Haggard, W.O. (2013) A Daptomycin-Xylitol-Loaded Polyme- 
thylmethacrylate Bone Cement: How Much Xylitol Should Be Used? Clinical Orthopaedics and Related Research, 471, 3149-3157. http://dx.doi.org/10.1007/s11999-013-2939-1

[26] Frutos, G., Pastor, J.Y., Martínez, N., Virto, M.R. and Torrado, S. (2010) Influence of Lactose Addition to GentamicinLoaded Acrylic Bone Cement on the Kinetics of Release of the Antibiotic and the Cement Properties. Acta Biomaterialia, 6, 804-811. http://dx.doi.org/10.1016/j.actbio.2009.08.028

[27] Nugent, M., McLaren, A., Vernon, B. and McLemore, R. (2010) Strength of Antimicrobial Bone Cement Decreases with Increased Poragen Fraction. Clinical Orthopaedics and Related Research, 468, 2101-2106. http://dx.doi.org/10.1007/s11999-010-1264-1

[28] Liu, W.C., Wong, C.T., Fong, M.K., Cheung, W.S., Kao, R.Y.I., Luk, K.D.K. and Lu, W.W. (2010) Gentamicin-Loaded Strontium-Containing Hydroxyapatite Bioactive Bone Cement-An Efficient Bioactive Antibiotic Drug Delivery System. Journal of Biomedical Materials Research Part B: Applied Biomaterials, 95B, 397-406. http://dx.doi.org/10.1002/jbm.b.31730

[29] Shen, S.C, Ng, W.K., Shi, Z., Chia, L., Neoh, K.G. and Tan, R.B.H. (2011) Mesoporous Silica Nanoparticle-Functionalized Poly (Methyl Methacrylate)-Based Bone Cement for Effective Antibiotics Delivery. Journal of Materials Science: Materials in Medicine, 22, 2283-2292. http://dx.doi.org/10.1007/s10856-011-4397-1

[30] Masri, B.A., Duncan, C.P., Beauchamp, C.P., Paris, N.J. and Arntorp, J. (1995) Effect of Varying Surface Patterns on Antibiotic Elution from Antibiotic-loaded Bone Cement. The Journal of Arthroplasty, 10, 453-459. http://dx.doi.org/10.1016/S0883-5403(05)80145-7

[31] Cai, X.Z., Chen, X.Z., Yan, S.G., Ruan, Z.R., Yan, R.J., Ji, K. and Xu, J. (2009) Intermittent Watt-Level Ultrasonication Facilitates Vancomycin Release from Therapeutic Acrylic Bone Cement. Journal of Biomedical Materials Research Part B: Applied Biomaterials, 90B, 11-17. http://dx.doi.org/10.1002/jbm.b.31288

[32] Shiramizu, K., Lovric, V., Leung, A. and Walsh, W.R. (2008) How Do Porosity-Inducing Techniques Affect Antibiotic Elution from Bone Cement? An Intro Comparison between Hydrogen Peroxide and a Mechanical Mixer. Journal of Orthopaedics and Traumatology, 9, 17-22. http://dx.doi.org/10.1007/s10195-008-0099-y

[33] Beenken, K.E., Bradney, L., Bellamy, W., Skinner, R.A., McLaren, S.G., Gruenwald, M.J., Spencer, H.J., Smith, J.K., Haggard, W.O. and Smeltzer, M.S. (2012) Use of Xylitol to Enhance the Therapeutic Efficacy of Polymethylmethacrylate-Based Antibiotic Therapy in Treatment of Chronic Osteomyelitis. Antimicrobial Agents and Chemotherapy, 56, 5839-5844. http://dx.doi.org/10.1128/AAC.01127-12

[34] Klekamp, J., Dawson, J.M., Haas, D.W., DeBoer, D. and Christie, M. (1999) The Use of Vancomycin and Tobramycin in Acrylic Bone Cement: Biomechanical Effects and Elution Kinetics for Use in Joint Arthroplasty. The Journal of Arthroplasty, 14, 339-346. http://dx.doi.org/10.1016/S0883-5403(99)90061-X

[35] Frutos, P., Diez-Peña, E., Frutos, G. and Barrales-Rienda, J.M. (2002) Release of Gentamicin Sulphate from a Modified Commercial Bone Cement. Effect of (2-Hydroxyethyl Methacrylate) Comonomer and Poly ( $N$-vinyl-2-pyrrolidone) Additive on Release Mechanism and Kinetics. Biomaterials, 23, 3787-3797. http://dx.doi.org/10.1016/S0142-9612(02)00028-5

[36] American Society for Testing and Materials (ASTM) (2011) Standard F 2118-10 (Approved December 1, 2010): Standard Test Method for Constant Amplitude of Force Controlled Fatigue Testing of Acrylic Bone Cement Materials. ASTM International, West Conshohocken.

[37] Richelsoph, K.C., Webb, N.D. and Haggard, W.O. (2007) Elution Behavior of Daptomycin-Loaded Calcium Sulfate Pellets: A Preliminary Study. Clinical Orthopaedics \& Related Research, 461, 68-73.

[38] Korsmeyer, R.W., Gurny, R., Doelker, E., Buri, P. and Peppas, N.A. (1983) Mechanisms of Solute Release from Porous Hydrophilic Polymers. International Journal of Pharmaceutics, 15, 25-35. http://dx.doi.org/10.1016/0378-5173(83)90064-9

[39] Lindner, W.D. and Lippold, B.C. (1995) Drug Release from Hydrocolloid Embedding with High or Low Susceptibility to Hydrodynamic Stress. Pharmaceutical Research, 12, 1781-1785. http://dx.doi.org/10.1023/A:1016238427313

[40] Hesaraki, S., Moztarzadeh, F., Nemati, R. and Nezafati, N. (2009) Preparation and Characterization of Calcium Sulfate-Biomimetic Apatite Nanocomposites for Controlled Release of Antibiotics. Journal of Biomedical Materials Research Part B: Applied Biomaterials, 91B, 651-661. http://dx.doi.org/10.1002/jbm.b.31441

[41] Noyes, A.A. and Whitney, W.R. (1897) The Rate of Solution of Solid Substances in Their Own Solutions. Journal of the American Chemical Society, 19, 930-934. http://dx.doi.org/10.1021/ja02086a003

[42] Anagnostakos, K. and Kelm, J. (2009) Enhancement of Antibiotic Elution from Acrylic Bone Cement. Journal of Biomedical Materials Research Part B: Applied Biomaterials, 90B, 467-475. http://dx.doi.org/10.1002/jbm.b.31281

[43] Lewis, G. (2009) Properties of Antibiotic-Loaded Acrylic Bone Cements for Use in Cemented Arthroplasties: A State -of-the-Art Review. Journal of Biomedical Materials Research Part B: Applied Biomaterials, 89B, 558-574. http://dx.doi.org/10.1002/jbm.b.31220 
[44] Siepmann, J. and Peppas, N.A. (2001) Modeling of Drug Release from Delivery Systems Based on Hydroxypropyl Methylcellulose (HPMC). Advanced Drug Delivery Reviews, 48, 139-157.

http://dx.doi.org/10.1016/S0169-409X(01)00112-0

[45] Kühn, K.D. (2000) Bone Cements: Up-to-Date Comparison of Physical and Chemical Properties of Commercial Materials. Springer-Verlag, Berlin. http://dx.doi.org/10.1007/978-3-642-59762-6

[46] Mattila, P.T., Svanberf, M.J., Jämsä, T. and Knuuttila, M.L.E. (2002) Improved Bone Biomechanical Properties in Xylitol-Fed Aged Rats. Metabolism, Clinical and Experimental, 51, 92-96. http://dx.doi.org/10.1053/meta.2002.28105

[47] Gálvez-Lopez, R., Peña-Monje, A., Antelo-Lorenzo, R., Guardia-Olmedo, J., Moliz, J., Hernández-Quero, J. and ParraRuiz, J. (2014) Elution Kinetics, Antimicrobial Activity, and Mechanical Properties of 11 Different Antibiotic Loaded Acrylic Bone Cement. Diagnostic Microbiology \& Infectious Disease, 78, 70-74.

http://dx.doi.org/10.1016/j.diagmicrobio.2013.09.014 